\section{International Scientific Journal Theoretical \& Applied Science}

p-ISSN: 2308-4944 (print) $\quad$ e-ISSN: 2409-0085 (online)

Year: $2015 \quad$ Issue: 11 Volume: 31

Published: $30.11 .2015 \quad$ http://T-Science.org

SECTION 27. Transport.
Andrey Stanislavovich Reshenkin professor candidate of technical sciences, head of department

Don State Technical University, Russia v.serge.79@mail.ru

Sergey Sergeevich Vorobyev assistant professor candidate of technical sciences Don State Technical University, Russia

Dmitriy Sergeevich Andros student Don State Technical University, Russia

Victor Gennad'evich Babkin student

Don State Technical University, Russia

Andrey Yur'evich Ganchurin student

Don State Technical University, Russia

\title{
LOGISTIZATION OF THE ENTERPRISES OF THE MAIL SERVICE ON THE BASIS OF THE MODEL OF DIFFERENTIATION OF SERVICE ZONES
}

Abstract: In the given article we have considered the purpose and the mechanism of logistization of communications enterprises "Mail of Russia". The model of differentiation of service zones of regional post offices is offered. The model is based on the calculation of lines of equal costs for delivery of mail to the neighboring post offices. Such approach allows optimizing the costs of post offices service and, as a result, to increase the efficiency of functioning of the branch "Mail of Russia" in Rostov region.

Key words: logistization, model, optimization, efficiency of functioning.

Language: Russian

Citation: Reshenkin AS, Vorobyev SS, Andros DS, Babkin VG, Ganchurin AY (2015) LOGISTIZATION OF THE ENTERPRISES OF THE MAIL SERVICE ON THE BASIS OF THE MODEL OF DIFFERENTIATION OF SERVICE ZONES. ISJ Theoretical \& Applied Science 11 (31): 161-170.

Soi: http://s-o-i.org/1.1/TAS-11-31-26 Doi: crossef http://dx.doi.org/10.15863/TAS.2015.11.31.26

\section{ЛОГИСТИЗАЦИЯ ПРЕДПРИЯТИЙ ПОЧТОВОЙ СВЯЗИ НА ОСНОВЕ МОДЕЛИ РАЗГРАНИЧЕНИЯ ЗОН ОБСЛУЖИВАНИЯ}

Аннотация: В статье рассмотрены цель и механизм логистизации предприятий связи "Почтьл России". Предложена модель разграничения зон обслужсивания районных почтамтов. В основу модели положен расчет линий равных затрат на доставку почтовых отправлений в соседние почтамты. Такой подход позволяет оптимизировать затраты на обслуживание почтовых отделений связи и как следствие - повысить эффективность функиионирования филиала "Почты России" в Ростовской области.

Ключевые слова: логистизация, модель, оптимизация, эффективность функционирования.

Логистика, как и маркетинг, является научно-практическим инструментом совместного хозяйствования многих экономически самостоятельных рыночных структур [1]. С помощью современных форм и методов логистики, возможно, достигать рациональной (оптимальной) организации потоковых процессов, протекающих в пространственновременной последовательности, с целью выявления и реализации потенциальных резервов управления и получения, в конечном счете, дополнительных доходов и прибыли этими структурами преимущественно за счет 
общественно-полезных, главным образом, производительных факторов и источников [2].

В условиях повышения конкуренции на региональном почтовом рынке вопросы повышения качества оказания почтовых услуг, связанных с перемещением почтовых отправлений (ПО) от отправителя к адресату приобретают особую актуальность. Как отмечают специалисты, основная составляющая качества в почтовой связи обусловлена скоростью прохождения ПО и обеспечением их сохранности. Выполнение этих показателей зависит от правильной организации перевозки почты автомобильным транспортом [3]. Вместе с тем, с позиции логистики повышение качества предполагает сокращение времени выполнения основных логистических операций, входящих в производственный цикл почтового производства. К ним относятся: приём ПО, их обработка, транспортировка и доставка.

Из экономической теории известно, что почтовое производство, относимое к материальному производству, обладает особенностью, суть которого состоит в том, что его конечным результатом является процесс оказания услуги по доставке почтовых отправлений от отправителя к адресату в заданные сроки, с требуемой степенью безопасности. При этом каждое почтовое отправление в своем движении от отправителя до получателя проходит ряд обязательных технологических этапов единого почтового технологического процесса, в результате которого заказ на услугу почтовой связи превращается в продукцию почтовой связи, физической сущностью которой является полезный эффект перемещения.

В данной работе, учитывая мнения таких отечественных учёных, как Стаханова В.И., Украинцева В.Б о том, что в «потоковых процессах воспроизводства и обращения, происходящих в условиях либерализации рынка возникают естественные противоречия и различные группы проблем, которые в значительной степени могут сглаживаться или быть решены при логистизации потоковых процессов обращения» [4], а также то, что «логистизация материальных потоков рациональная организация и управление ими, которые ... п предполагают обязательное использование основных логистических принципов однонаправленности, гибкости, синхронизации, оптимизации, интеграции потоковых процессов [5] нами рассматривается возможность осуществления логистизации потоковых процессов ПО, циркулирующих между районными почтовыми почтамтами (РП) Управления федеральной почтовой связи по Ростовской области (УФПС РО) - филиала ФГУП «Почта России» по Ростовской области.

Согласно классификации, приведенной в [6] почтовая система УФПС РО относиться к макрологистическим системам, для которых в настоящее время основной тенденцией является глобализация.

Целью макрологистической системы УФПС PO, структурная схема которой представлена на рисунке 1, является доставка почтовых отправлений в заданное место, в нужное время, в требуемом количестве при минимальном уровне совокупных издержках.

Как видно из рисунка 1, общее количество районных почтовых почтамтов (РП) составляет 50 шт., которые по своему функционально логистическому содержанию являются основными функциональными звеньями в макрологистической системы УФПС РО, от качества, функционирования которых в конечном итоге зависит эффективность работы всей почтовой сети УФПС РО. Критерием эффективности почтового производства в условиях рынка является величина получаемого УФПС РО совокупного дохода, которая в свою очередь, как показывает практика, зависит от величины совокупных логистических издержек.

Исследуя вопросы логистизации предприятий связи УФПС РО, структурная схема которой представлена на рисунок 1 , необходимо обратить особое внимание на то, что цель почтовой логистизации совпадает с теми основными целями логистизации, которые указаны видным специалистом по логистики Е.К. Ивакиным. Это - сокращение потерь несопряжённости потоков, повышение качества и конкурентоспособности, повышение адаптивности производства к изменяющейся конъюнктуре рынка [7]. 


\begin{tabular}{|c|c|c|c|c|c|c|}
\hline Impact Factor: & $\begin{array}{l}\text { ISRA (India) } \\
\text { ISI (Dubai, UAE } \\
\text { GIF (Australia) } \\
\text { JIF }\end{array}$ & $\begin{array}{l}=1.344 \\
=0.829 \\
=0.564 \\
=1.500\end{array}$ & $\begin{array}{l}\text { SIS (USA) } \\
\text { PИНЦ (Russia) } \\
\text { ESJI (KZ) } \\
\text { SJIF (Morocco) }\end{array}$ & $\begin{array}{l}=0.912 \\
=0.179 \\
=1.042 \\
=2.031\end{array}$ & $\begin{array}{l}\text { ICV (Poland) } \\
\text { PIF (India) }\end{array}$ & $\begin{array}{l}=6.630 \\
=1.940\end{array}$ \\
\hline
\end{tabular}

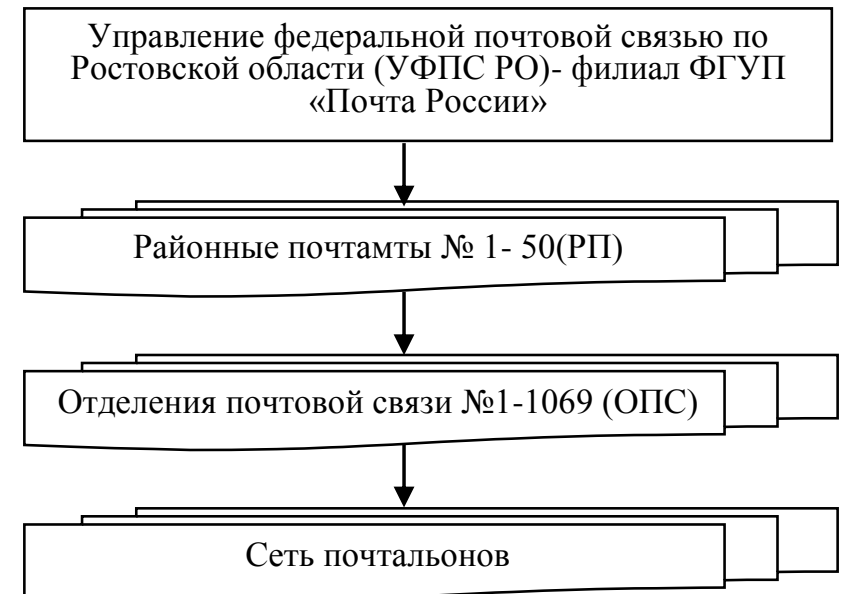

Рисунок 1 - Структурная схема почтовой сети УФПС РО.

Кроме того, в дополнение к вышеизложенному, из теории известно, что логистизация позволяет представлять управляемый процесс в виде потока или формировать потоковую модель и осуществлять оптимизацию траектории и параметров потока, а значит и всей производственно- коммерческой деятельности УФПС РО [8].

Таким образом, в данной работе, нами при исследовании процесса логистизации предприятий связи УФПС РО было учтено мнение видных отечественных учёных Альбекова А.У., Ивакина Е.К., Сергеева В.И и Стаханова В.Н.о том, что только на основании выполнения логистизации можно осуществлять разработку концепции развития предприятия, а так же формировать логистические цепи и системы.

Актуальность исследования вопросов логистизации предприятий связи приобретает наибольшую актуальность в настоящее время, в связи с переходом от рынка продавца к рынку покупателя, который требует от почтовой связи гибкого реагирования почтовых производственных систем и почтового транспорта на быстро изменяющиеся приоритеты потребителей почтовой продукции [9].

С учётом этого, применительно к почтовой сети УФПС РО процесс логистизации её управляющих воздействий можно представить в виде блок - схемы (рисунок 2).

Структурный анализ блочный схемы, представленной на рисунке 2 позволяет сделать вывод о том, что усиление общего эффекта работы системы управления УФПС РО или синергетика логистики связано с процессом оптимизации этих воздействий.

Математически вышеизложенное, может быть описано в следующем виде [8]:

$$
\text { opt } \left.\sum_{i=1}^{m} X_{i}\right\rangle \sum_{i=1}^{m} \text { opt } X_{i}
$$

или

$$
\text { opt } \sum_{i=1}^{m} X_{i}-\sum_{i=1}^{m} \text { opt } X_{i}=\Delta
$$

где, $\Delta-$ синергетический эффект логистики; $1,2, \ldots \mathrm{I} . . . m$-звенья логистической цепи;

$X_{i}$ - показатель оптимальности і-го звена

Причём данная оптимизация должна касаться суммы (совокупности) воздействий или всего процесса в целом, т.е. «оптимум суммы», а не каждого в отдельности воздействия - «суммы оптимумов». 


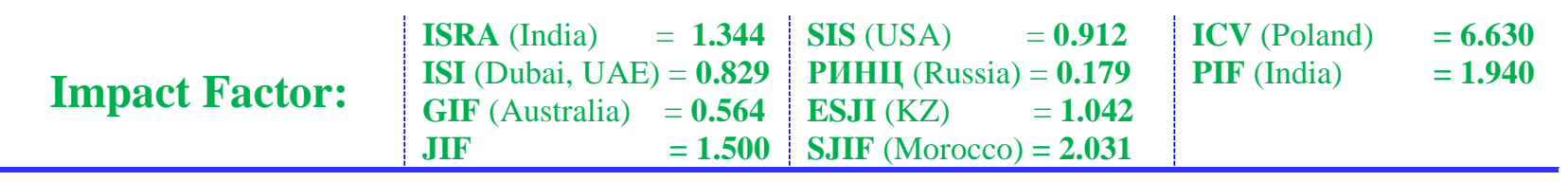

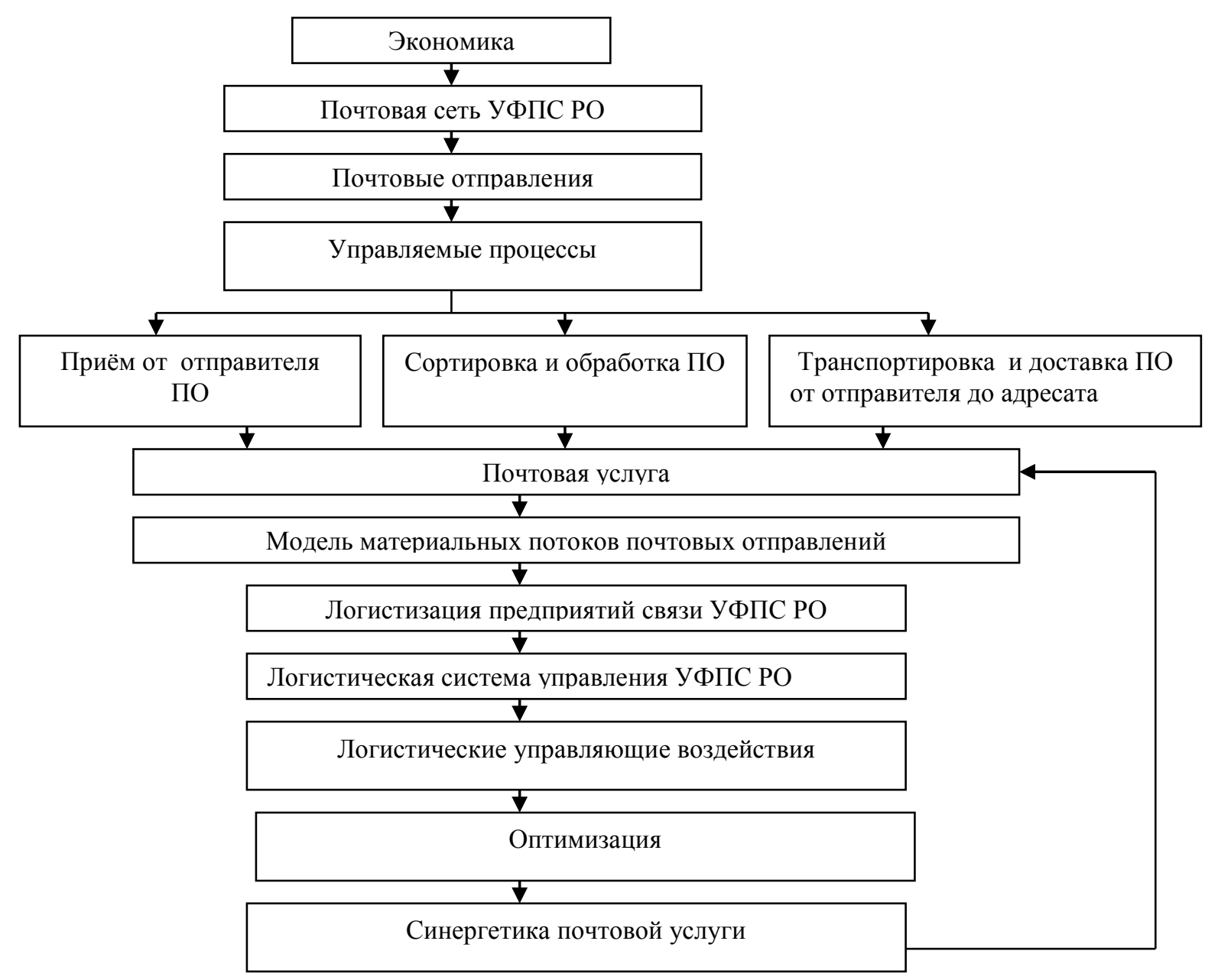

Рисунок 2 - Логистизация управляемых процессов в почтовой сети УФПС РО.

В этом случае, рассмотрение $о p t \sum_{i=1}^{m} X_{i}$ предполагает в качестве объекта оптимизации использование всей совокупности управляющих воздействий в масштабе УФПС РО. Это полностью соответствует основным методологическим положениям, предложенным Дж. Гигом [10] и отечественными учёными Стахановым В.Н. и Ивакиным Е.К.[11], согласно которым улучшение логистической системы можно осуществить, как путём трансформации традиционных систем управления экономическими потоками в логистические, так и совершенствование уже имеющихся логистических систем.

Как отмечалось ранее, осуществление почтовой услуги по своей физической природе является перемещение почтовых отправлений от отправителя до адресата в виде потокового процесса.
Поэтому полную логистическую цепь в почтовой отрасли можно представить в виде $\Pi \rightarrow \mathrm{P} \rightarrow \mathrm{Y}$,

где П - почтовые отправления;

Р-почтовое производство;

$Y$-потребление адресатом почтовой продукции.

Анализируя физическую природу почтовой связи, основу которой составляет качественное осуществление почтовой услуги по перемещению почтовых отправлений от отправителя до адресата в требуемые сроки, можно сказать, что почтовую связь необходимо рассматривать, как потоковый процесс, к которому применим классический логистический подход.

Тогда, принимая во внимание, что спрос на почтовую продукцию в большей степени зависит от отправителя почтовых отправлений, можно считать, что имеет место следующая функциональная зависимость:

$$
Y=f(Q),
$$


где: Y- потребление адресатом почтовой продукции, $Q$ - объём почтовых отправлений.

Раскрывая структурно-экономическое содержание зависимости $Y=f(Q)$ получаем схему логистической цепи УФПС РО, представленной на рисунке 3 .

Из рисунка 3 видно, что создаваемые почтовые потоки по каждому виду отправлений при своём движении от отправителя к адресату проходят ряд обязательных этапов единого производственного процесса, в результате которого заказ на почтовую услугу превращается в почтовую продукцию, сущность которого является перемещение.

Данный единый производственный процесс организационно разбивается на комплекс производственных операций (приём, обработка, сортировка, перевозка, доставка), обеспечивающих подготовку принятых от клиентов или, поступающих от других предприятий связи почтовых отправлений к пересылке по назначению и вручению [12].

Практическое осуществление такого единого производственного почтового процесса предполагает логистическую организацию почтовоматериальных и сопутствующих им потоков, которое должно основываться на следующих принципах [6]:

-оптимизации и рационализации транспортно-экономических связей, направлений и структуры материальных и других видов экономических оттоков;

-минимизации совокупных затрат в полной логистической цепи от отправителя до получателя;

-удовлетворение потребности в почтовой услуге в нужное время, в нужном месте, в заданном объёме осуществляется в определённых хозяйственных системах (региональных, районных) и согласованных с общими целями их развития.

Приведенная логистическая цепь осуществления почтовой услуги УФПС РО предполагает целостность единого технологического процесса почтового производства, в котором доминантная роль отведена РП. При этом одним из основных показателей, характеризующих работу данной логистической цепи, является почтовый обмен число почтовых отправлений принятых, обработанных и переданных по назначению за определённый интервал времени.

В зависимости от источника поступления ПО, их направления перемещения различают:
- исходящий обмен - принятый от клиентуры и подготовленный к пересылке по назначению;

- входящий - поступающие ПО на предприятия связи для вручения адресатам;

-транзитный - поступающие ПО на предприятия связи для обработки и дальнейшей пересылки по назначению.

В почтовой связи принято, что входящий обмен равен исходящему [13], однако для отдельных предприятий связи величина и структура их могут различными.

Характерными свойствами почтовых потоков является неравномерность по времени и направлениям, а также неопределённость реального состояния внешней среды в связи с тем, что ряд её факторов имеет стохастический характер (число почтовых отправлений, поступающих на предприятия связи, расписание движения транспорта и его изменение, дорожноклиматические условия). Это увеличивает себестоимость обработки и транспортировки почтовых отправлений.

C позиции логистического подхода необходимо отметить, что каждый вид почтового обмена определяет отдельные почтовые потоки, которые определяются числом почтовых отправлений одного вида, которые следует в определённом направлении.

Причём структура, свойства и количественные характеристики почтовых потоков имеют первостепенное значение для рационального построения всей почтовой системы УФПС РО.

Следует отметить, что неравномерность во времени обусловлена неравномерностью приёма корреспонденции от населения, неравномерностью в движении транспорта, сезонными колебаниями спроса на услуги почтовой связи.

Для решения данного вопроса увеличивают частоту выемку писем из почтовых ящиков, а также частоту объездов по почтовым маршрутам, проводят рекламу почты по телевидению.

Рассматривая почтовое производство, необходимо подчеркнуть важность работы районных почтамтов, силами которых производиться приём ПО, забираемых из закреплённых за ним почтовых отделений, которые затем обрабатываются в РП, и доставляется в соответствии с указанным адресом на ПО в почтовые отделения, для доставки адресату или для дальнейшей обработке в зональный сортировочный центр (хаб) для дальнейшей пересылки в магистральной цепи. 


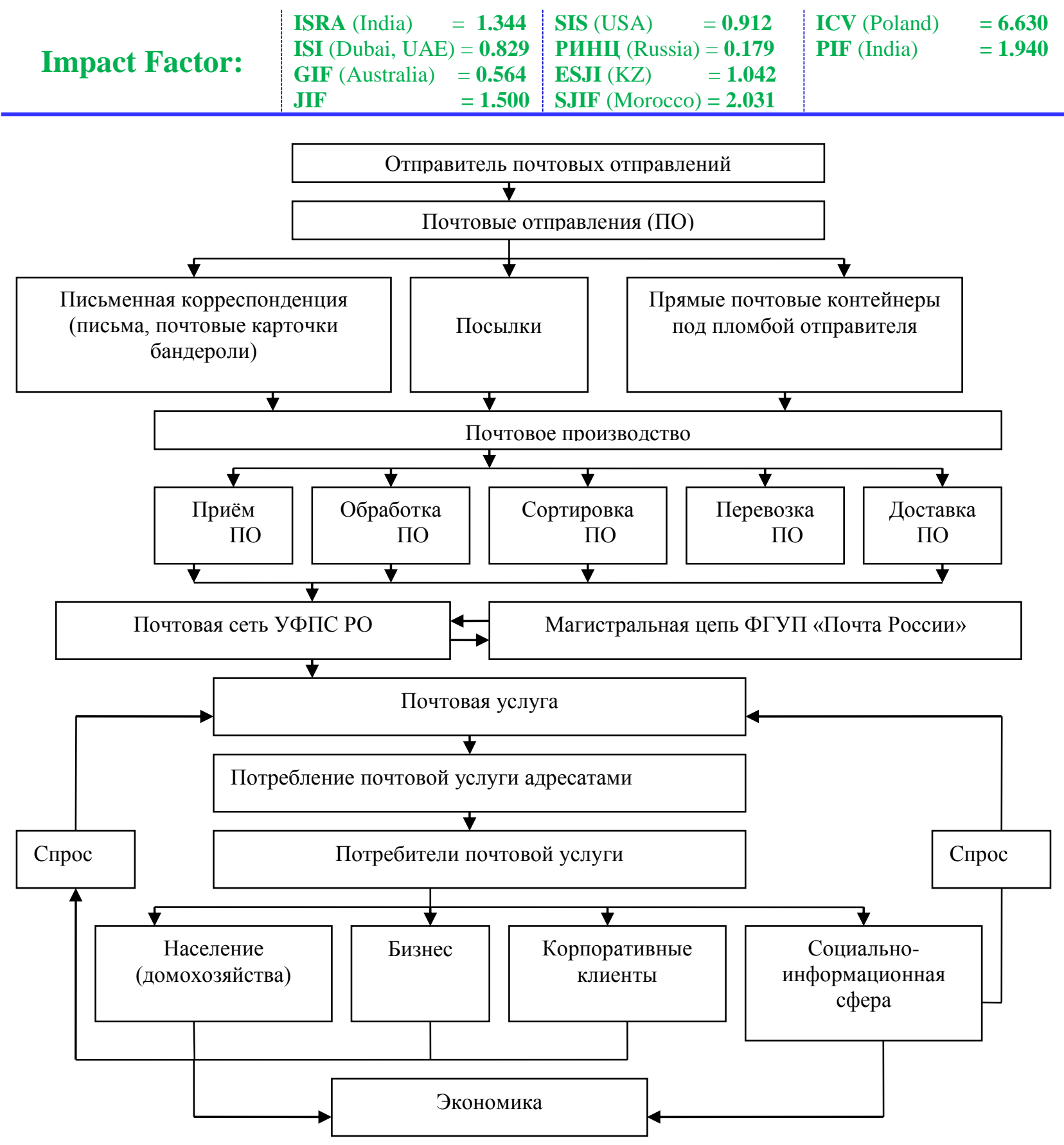

Рисунок 3 - Логистическая цепь осуществления почтовой услуги.

При рассмотрении вопроса логистизации предприятий связи УФПС РО нами принимается, что процесс формирования логистических операций не выполним без реализации такой системной функции логистики, как обеспечение сквозного движения материального почтового потока от отправителя до адресата. Для организации и контроле такого движения необходимо знать начальное состояние материального потока и то, как оно преобразуется под воздействием окружающей среды в потребительский продукт, попутно контролировать и регулировать логистические издержки, для чего нужно знать закономерности сохранения потребительских свойств (операции, сопровождающие процессы обмена, хранения, транспортировки, складирования, контроля и т.д.)

На почте к числу источников материальных потоков в виде ПО можно отнести 1069 почтовые отделения УФПС РО, в то время как основными функциональными звеньями являются 50 РП. На РП происходит формирование большей доли логистических издержек, так как силами и средствами РП осуществляются процессы перевозки ПО, в то время, как отмечают специалисты расходы на перевозку занимают54\% всех затрат [14].

При этом, как отмечают ведущие отечественные и зарубежные ученые и 
специалисты, под транспортировкой следует понимать комплексную логистическую функцию, предполагающую совокупность процессов, не только таких как погрузка- разгрузка, экспедирование, а также маршрутизацию перевозок и определение рациональных, а лучше оптимальных маршрутов [15].

Поэтому, если в соответствии с системным подходом и согласно [16] рассматривать районные почтамты, как « центры сосредочения затрат», которые несут ответственность за всю массу затрат, приходящийся на данный центр, то «пунктом сосредочения затрат» или наиболее затратным участком для РП является участок транспортировки. В связи с этим перед каждым РП и УФПС РО стоит задача анализа совершенствования единого технологического процесса на наиболее затратных участках, т.е. на участках транспортировки ПО.

При анализе деятельности районных почтамтов нами делается следующее ограничение- рассматриваются только внутренние материальные потоки ПО, которые в свою очередь, согласно установленной почтовой классификации подразделяются на исходящие и входящие, т.е. нами исследуются те грузопотоки ПО, которые циркулируют по территории Ростовской области, составляющей 97 км $^{2}$ и за организацию которых несёт ответственность УФПС РО.

При этом следует учитывать, что почтовая сеть УФПС РО охватывает всю территория Ростовской области, которая поделена на следующие административно- территориальные единицы: города -23 , в том числе города областного подчинения-16, города районного подчинения -7, посёлки городского типа - 25, сельские районы - 43, сельские населённые пункты - 2297 [17].

Для решения поставленной задачи по исследованию возможностей логистизации процессов функционирования РП воспользуемся методом математического моделирования. С помощью которого, создав модель разграничения зон обслуживания районных почтамтов исследуем возможности уменьшения логистических издержек на «пунктах сосредочения затрат», связанных с транспортировкой ПО районными почтамтами.

В основу предлагаемого модели нами предлагается инновационный принцип создания зон обслуживания вокруг каждого из РП, который предполагает использование системного подхода, заключающийся в такой организации перевозочных процессов, которая предполагает рационализацию и оптимизацию почтовых маршрутов доставки почтовых отправлений в направлении минимизации совокупных затрат, как для РП, так и для УФПС по РО.

При этом нами учитывается такие особенности почтовой сети УФПС РО, как её протяженность и разветвлённость охвата всей территории Ростовской области, а также то, что это большая сложная система, состоящая из 50 районных почтамтов (РП) и 1069 отделения почтовой связи (ОПС), и в основе её системы управления лежит иерархический принцип управления.

Кроме того, нами учитывалось то, что размещение основных звеньев данной почтовой сети, а именно районных почтамтов, осуществлялось в экономической системе СССР по административно - территориальному принципу. А в настоящее время для УФПС РО экономическая целесообразность, усиленная влиянием почтового рынка (конкуренция, факторы глобализации) требует пересмотра, существующего экономически невыгодного принципа отнесения ОПС к тому или иному РП.

Таким образом, положительное решение задачи маршрутизации позволит реализовать принцип сквозного управления «УФПС РО - РП - ОПС», что в свою очередь будет способствовать развитию механизмов универсального обслуживания (предоставление необходимого набора услуг связи в заданный срок, с установленным качеством, по доступной цене), а также совершенствовать систему управления издержками каждого предприятий связи, тем самым, повышая их конкурентоспособность.

Учитывая, что конкурентность предприятий связи и УФПС РО в целом определяется их способностью управлять своими издержками нами предлагается экономико - математическая модель разграничения зон обслуживания районных почтамтов, которая позволяет на основании графического анализа линий равных затрат на доставку почты получить оптимальные зоны обслуживания почтовых отделений связи тем или иным районным почтамтом.

Рассмотрим систему доставки почты состоящую из двух районных почтамтов и главного почтамта.

В декартовой системе координат (рисунок 4) главный почтамт обозначен буквой Ц и имеет координаты $(0 ; 0)$, районные почтамты обозначены А и В с координатами $\left(\mathrm{x}_{\mathrm{a}} ; \mathrm{y}_{\mathrm{a}}\right)$ и $\left(\mathrm{x}_{\mathrm{b}} ; \mathrm{y}_{\mathrm{b}}\right)$ соответственно. При этом главный почтамт находится на расстояниях $\mathrm{a}$ и $\mathrm{b}$ от районных почтамтов. 


\begin{tabular}{|c|c|c|c|c|c|c|}
\hline Impact Factor: & $\begin{array}{l}\text { ISRA (India) } \\
\text { ISI (Dubai, UAE } \\
\text { GIF (Australia) } \\
\text { JIF }\end{array}$ & $\begin{array}{l}=1.344 \\
=0.829 \\
=0.564 \\
=1.500\end{array}$ & $\begin{array}{l}\text { SIS (USA) } \\
\text { PИНЦ (Russia) } \\
\text { ESJI (KZ) } \\
\text { SJIF (Morocco) }\end{array}$ & $\begin{array}{l}=0.912 \\
=0.179 \\
=1.042 \\
=2.031\end{array}$ & $\begin{array}{l}\text { ICV (Poland) } \\
\text { PIF (India) }\end{array}$ & $\begin{array}{l}=6.630 \\
=1.940\end{array}$ \\
\hline
\end{tabular}

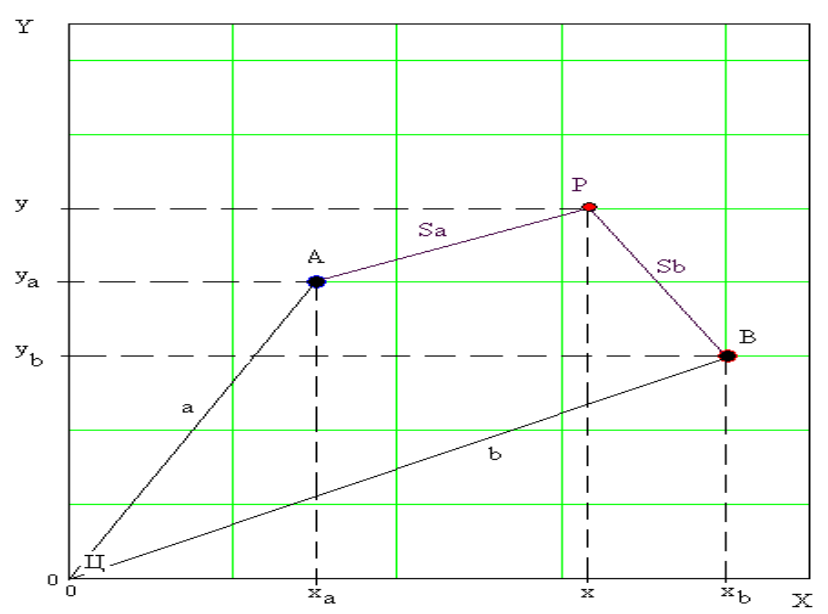

Рисунок 4 - Система доставки почты состоящая из двух районных почтамтов и главного почтамта.

Найдем кривую равных затрат на доставку почты через районные почтамты из центра.

Пусть точка $P(x ; y)$ лежит на искомой зависимости. Тогда цена доставки из районных почтамтов определяется по выражениям:

$$
\boldsymbol{Ц}_{A P}=C_{A} \boldsymbol{S}_{A}, \quad Ц_{B P}=C_{B} S_{B},
$$

где $S_{A}$ и $S_{B}$ расстояния от районных почтамтов А и $\mathrm{B}$ до точки $\mathrm{P}$, a $\mathrm{C}_{\mathrm{A}}$ и $\mathrm{C}_{\mathrm{B}}$ средние себестоимости доставки почты их центра Ц в точку $\mathrm{P}$ через районные почтамты А и В соответственно.

Исходя из условия равенства затрат приравняем правые части выражений (1).

$$
\mathrm{C}_{\mathrm{A}} \mathrm{S}_{\mathrm{A}}=\mathrm{C}_{\mathrm{B}} \mathrm{S}_{\mathrm{B}}
$$

Возведем левую и правую части в квадрат и разрешим относительно $S_{A}$.

$$
\begin{aligned}
S_{A}^{2} & =\left(\frac{C_{B}}{C_{A}}\right)^{2} \cdot S_{B}^{2} \\
k & =\left(\frac{C_{B}}{C_{A}}\right)^{2} \cdot \quad \text { Тогда } \\
S_{A}^{2} & =k \cdot S_{B}^{2} .
\end{aligned}
$$

Подставим в (4) квадраты расстояний, $S_{A}{ }^{2} S_{B}^{2}$ выраженные через координаты (5).

$$
\begin{aligned}
& S_{A}^{2}=\left(y-y_{A}\right)^{2}+\left(x-x_{A}\right)^{2} \\
& S_{B}^{2}=\left(y-y_{B}\right)^{2}+\left(x-x_{B}\right)^{2}
\end{aligned}
$$

После раскрытия скобок, приведения подобных разделим полученное уравнение на (1k).
С учетом, что

$$
\begin{aligned}
& x_{A}^{2}+y_{A}^{2}=a^{2}, x_{B}^{2}+y_{B}^{2}=b^{2}, \text { a } \\
& \frac{2 \cdot\left(k \cdot y_{B}-y_{A}\right)}{1-k}=m \text { и } \frac{2 \cdot\left(k \cdot x_{B}-x_{A}\right)}{1-k}=n \text { получим } \\
& y^{2}+m \cdot y+n \cdot x+x^{2}=\frac{k \cdot b^{2}-a^{2}}{1-k} \\
& \text { Обозначив, } \quad x^{2}+n \cdot x-\frac{k \cdot b^{2}-a^{2}}{1-k}=D
\end{aligned}
$$

приходим к обыкновенному квадратному уравнению $y^{2}+m \cdot y+D=0$, корни которого являются искомой зависимостью.

На рисунке 5 представлены расчеты, где цифрами 1, 2 и 3 обозначены кривые, вычисленные при значениях коэффициентов $k=0.8, k=1.001$ и $k=1.2$ соответственно.

Полученные результаты показывают, что при заданных условиях расчета, кривые равных затрат разграничивают зоны нахождения подчиненных почтовых отделений.

Так, на рисунке 6 представлена модель для системы пяти (A, B, C, D, E) районных почтамтов и центра отправки (Ц) разграниченная линиями равных затрат на доставку почты. Полученные зоны могут являться условными территориями обслуживания почтовых отделений связи районными почтамтами.

Однако при практическом осуществлении логистического подхода в данном случае необходимо учитывать целый ряд дополнительных факторов. К ним следует отнести: развитие дорожной инфраструктуры; естественные природные препятствия т. п.[18]. 


\begin{tabular}{l|lrl|l|ll} 
& ISRA (India) & $=\mathbf{1 . 3 4 4}$ & SIS (USA) & $=\mathbf{0 . 9 1 2}$ & ICV (Poland) & $=\mathbf{6 . 6 3 0}$ \\
Impact Factor: & ISI (Dubai, UAE) $=\mathbf{0 . 8 2 9}$ & PUHU (Russia) $=\mathbf{0 . 1 7 9}$ & PIF (India) & $=\mathbf{1 . 9 4 0}$ \\
& GIF (Australia) & $\mathbf{0 . 5 6 4}$ & ESJI (KZ) & $=\mathbf{1 . 0 4 2}$ & & \\
& JIF & $=\mathbf{1 . 5 0 0}$ & SJIF (Morocco) $=\mathbf{2 . 0 3 1}$ & &
\end{tabular}

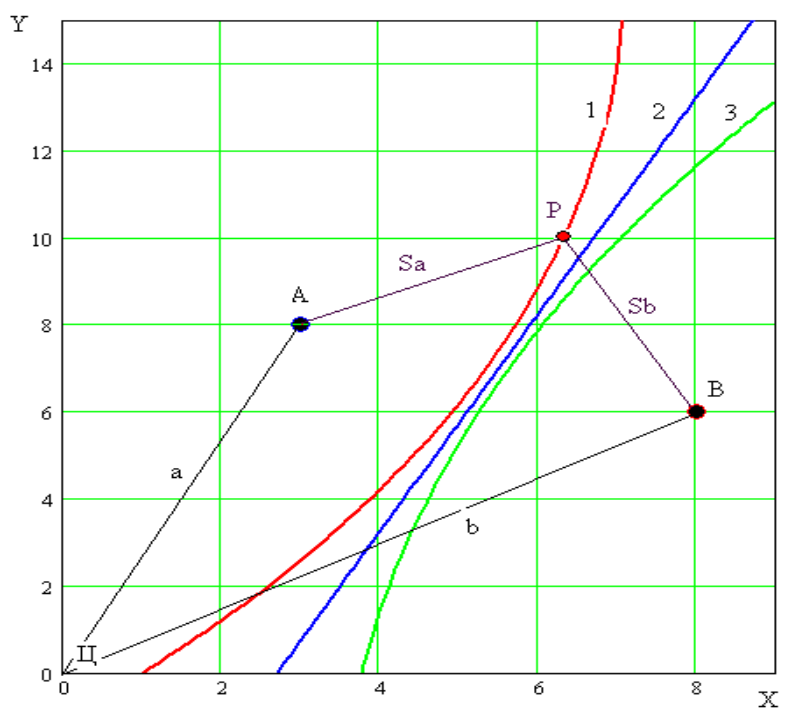

Рисунок 5 - Система из двух районных почтамтов.

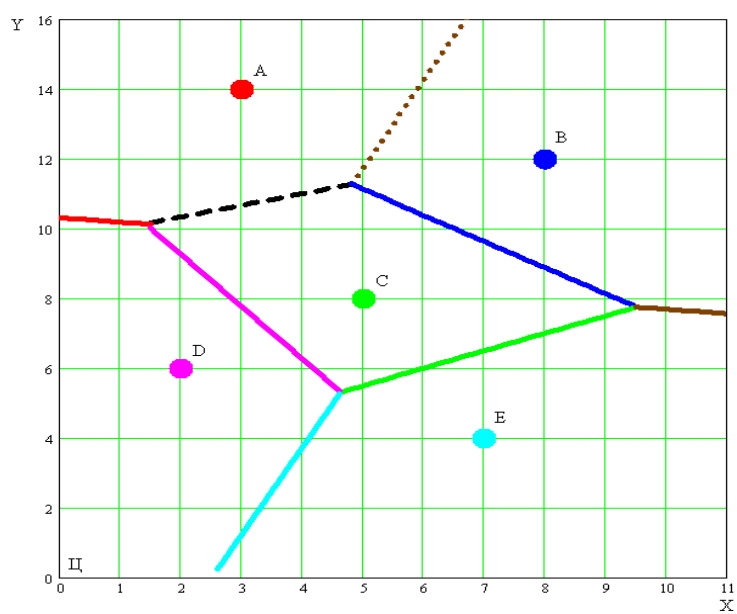

Рисунок 6 - Модель для системы пяти (A, B, C, D, E) районных почтамтов и центра отправки (Ц).

В заключении следует отметить, что на наш взгляд предложенная модель разграничения зон обслуживания районных почтамтов позволяет практически реализовать одно из возможных направлений снижения производственных логистических издержек путём организации производственного процесса в соответствии с его экономическим характером, а не в соответствии с раннее установленными традициями.

Кроме того, использование данной модели даёт возможность решить задачу анализа совершенствования единого технологического процесса в районных почтамтах на участках наибольших затрат, которыми являются участки транспортировки почтовой продукции. Это позволяет при выборе системы построения почтовых маршрутов учитывать особенности путей сообщения, достижения быстрейшего продвижения ПО, наиболее эффективно использовать транспортных средств и рационально расходовать денежных средств.

Использование предложенной модели предоставляет возможность учесть такие объективные ограничения, как ресурсная ограниченность УФПС РО и пространственновременные ограничения, накладываемые на перемещения почтовых потоков, что позволяет повысить высвобождаемую логистическую мощность традиционной почтовой системы УФПС РО в процессе её логистизации.

Резюмируя вышесказанное, можно сказать, что новая система позволит оптимизировать затраты на обслуживание почтовых отделений связи каждого почтамта и, как следствие, повысить эффективность функционирования УФПС РО - филиала ФГУП «Почта России». 


\begin{tabular}{l|lrl|l|ll} 
& ISRA (India) & $=\mathbf{1 . 3 4 4}$ & SIS (USA) & $=\mathbf{0 . 9 1 2}$ & ICV (Poland) & $=\mathbf{6 . 6 3 0}$ \\
Impact Factor: & ISI (Dubai, UAE) $=\mathbf{0 . 8 2 9}$ & PUHU (Russia) $=\mathbf{0 . 1 7 9}$ & PIF (India) & $=\mathbf{1 . 9 4 0}$ \\
& GIF (Australia) & $\mathbf{0 . 5 6 4}$ & ESJI (KZ) & $=\mathbf{1 . 0 4 2}$ & & \\
& JIF & $=\mathbf{1 . 5 0 0}$ & SJIF (Morocco) & $=\mathbf{2 . 0 3 1}$ & &
\end{tabular}

\section{References:}

1. Dzhabrailov AJ (2001) Teoreticheskie osnovy i principy logistizacii sfery transportno-skladskih uslug // Rossijskoe predprinimatel'stvo. 2001. — № 8 (20).

2. Semenenko AI, Sergeev VI (2003) Logistika. Osnovy teorii: Uchebnik dlja vuzov. - SPb.: Izdatel'stvo «Sojuz», 2003. - 544 p.

3. Zenkin NV (2004) Pochtovoe pravo. Pochtovye pravila /Instrukcija po organizacii perevozki pochty po pochtovym marshrutam, obsluzhivaemym avtomobil'nym i guzhevym transportom ot 9.05.1978, M.; Central'nyj izdatel'skij DOM, 2004.

4. Semenenko AV, Sergeev LB (2001) Logistika. Osnovy teorii: Uchebnik dlja vuzov, 2001.

5. Stahanov VN (2001) Teoreticheskie osnovy logistiki / V. N. Stahanov, V. B. Ukraincev. :Feniks, 2001. - 159 p.

6. Prokof'eva TA, Lopatkin OM (2003) Logistika transportno-raspredelitel'nyh sistem: Regional'nyj aspekt. M.; R. Konsul'tant, 2003.

7. Ivakin EK (1997) Logistika kapital'nogo stroitel'stva v regione. Rostov-na-Donu, RGSU, 1997.

8. Al'bekov AU, Tlepcerishhev AM (2002) Organizacija i funkcionirovanie logisticheskoj sistemy jenergeticheskogo kompleksa Rostovskoj oblasti: Monografija. RGJeU (RINH), Rostov - na - Donu, 2002.

9. Lukinskij VS (2000) Logistika avtomobil'nogo transporta: Koncepcii, metody, modeli. M.; Finansy i kredity, 2000.
10. Gig D (1981) Prikladnaja obshhaja teorija sistem. V 2-h kn. / Per. s ang. M.;Mir,1981.

11. Stahanov VN, Ivakin EK (1997) Logistika V stroitel'stve. Rostov-na-Donu, RGSU, 1997.

12. Verhova GV, et al. (2000) Tehnicheskie sredstva avtomatizacii pochtovoj svjazi: Uchebnik dlja vuzov, SPb, Politehnika, 2000.

13. Budenko VP, Mamzelev IA, et al. (1998) Tehnologicheskie processy v pochtovoj svjazi. Kn. 1. Osnovnye harakteristiki i tehnicheskoe obsluzhivanie. Uchebnik dlja vuzov. M.; Radio isvjaz', 1998.

14. (2003) Modeli i metody teorii logistiki./ Pod red. Lukinskogo L.B./. SPb, «Piter», 2003.

15. Karablin OV (2010) Jekonomika i tehnologija proizvodstva sistem i oborudovanija kompleksov/O.V. Karablin,A.S. Reshenkin. Rostov n/D, 2010.

16. Druker P (2003) Jeffektivnost' upravlenija. Jekonomicheskie zadachi i optimal'nye reshenija. / Per. s ang. M. Kotel'nikovoj. - M.; FAIR-PRESS, 2003.

17. (2014) Rostovskaja oblast' v cifrah 2013: Stat.sb./Rostovstat.- Rostov-na-Donu, 2014. $906 \mathrm{p}$.

18. (2014) Vvedenie v professional'nuju dejatel'nost'. Vorobyev S.S., Vorobyev S.A. (servis transportnyh sredstv) uchebnoe posobie / Pechataetsja po resheniju redakcionnoizdatel'skogo soveta Donskogo gosudarstvennogo tehnicheskogo universiteta. Rostov-na-Donu, 2014. 